\title{
Spectrally controlled interferometry for measurements of flat and spherical optics
}

Chase Salsbury, Artur G. Olszak

Chase Salsbury, Artur G. Olszak, "Spectrally controlled interferometry for measurements of flat and spherical optics," Proc. SPIE 10448, Optifab 2017, 104481C (16 October 2017); doi: 10.1117/12.2279811

SPIE. Event: SPIE Optifab, 2017, Rochester, New York, United States 


\title{
Spectrally controlled interferometry for measurements of flat and spherical optics
}

\author{
Chase Salsbury ${ }^{\mathrm{a}, \mathrm{b}}$ and Artur G. Olszak ${ }^{\mathrm{a}}$ \\ a ÄPRE Instruments, LLC, 2440 W Ruthrauff Rd 100, Tucson, USA \\ ${ }^{\mathrm{b}}$ College of Optical Sciences, University of Arizona, 1630 E University Blvd, Tucson, USA
}

\begin{abstract}
Conventional interferometry is widely used to measure spherical and flat surfaces with nanometer level precision but is plagued by back reflections. We describe a new method of isolating the measurement surface by controlling spectral properties of the source (Spectrally Controlled Interferometry - SCI). Using spectral modulation of the interferometer's source enables formation of localized fringes where the optical path difference is non-zero. As a consequence it becomes possible to form white-light like fringes in common path interferometers, such as the Fizeau. The proposed setup does not require mechanical phase shifting, resulting in simpler instruments and the ability to upgrade existing interferometers. Furthermore, it allows absolute measurement of distance, including radius of curvature of lenses in a single setup with possibility of improving the throughput and removing some modes of failure.
\end{abstract}

Keywords: Interferometry, spectrally controlled, white light, broadband, metrology, spherical, optics, coherence, radius of curvature, scanning interferometry

\section{INTRODUCTION}

Spectrally controlled interferometry is a novel approach to interferometry which benefits from combining many advantages of the two standard interferometry techniques: coherent ${ }^{1}$ and white-light. ${ }^{2}$ SCI utilizes modulation of the spectral distribution of the interferometer source to localize high contrast fringes at locations where the Optical Path Difference (OPD) does not equal to zero. Because fringe localization and phase modulation are implemented at the source level of the instrument, the system does not require mechanical assemblies for phase shifting nor path-length matching, simplifying the instrument without sacrificing measurement capability. Additionally, the spectral modulation implementation is electronically controlled and is capable of switching between coherent and incoherent (long and short coherence lengths, respectively) modalities of operation. This allows the system to be more easily aligned in coherent mode and then switched to incoherent mode to localize fringes. ${ }^{3}$ Due to controllable fringe localization, SCI is a natural solution to common interferometry problems such as the isolation of spurious back reflections from secondary surfaces which degrade measurement quality with phase print through and multiple beam interference fringes. Additionally, spectral modulation provides means of absolute distance assessment, the location of fringes relative to the reference surface is determined by the modulation parameters, hence this method can be also used to measure distance to the surface under test.

Kozhevatov, et al, ${ }^{4-6}$ and Schwider ${ }^{7,8}$ have previously established methods for producing localized fringes in unbalanced interferometers, with broadband sources through the use of a Fabry-Perot etalon. In both approaches, the etalon is used as a comb-like filter of the spectrum which produces a comb-like coherence function in OPD space. While localized fringes are produced at non-zero OPD locations, the fringe location is not unique and could lead to fringe ambiguity. Additionally, the fringe locations cannot be easily manipulated and phase shifting is still implemented with piezo-electric transducers. While the previously described methods and SCI are both able to produce localized fringes at non-zero OPD locations, SCI has the advantage of electronically controlled fringe location tuning and phase shifting, removing the need for mechanical path length matching and mechanical phase shifting.

Further author information: (Send correspondence to C. Salsbury)

C. Salsbury: E-mail: csalsbury@apre-inst.com, Telephone: +1-520-639-8195

A.G. Olszak: E-mail: aolszak@apre-inst.com

Optifab 2017, edited by Julie L. Bentley, Sebastian Stoebenau, Proc. of SPIE Vol. 10448, 104481C · @ 2017 SPIE · CCC code: 0277-786X/17/\$18 · doi: 10.1117/12.2279811 
Interferometric measurements of optical components are segmented into two standard categories: plano (flat) and spherical surfaces. For planar components with little to no wedge error, the reflections from each additional surface can add coherently at the detector, preventing accurate measurement of any one surface. ${ }^{9}$ While SCI has been demonstrated to address this challenge in the measurement of thin optical windows, ${ }^{3}$ we present SCI implemented with larger cavity distances and multiple surface cavities with maintained surface isolation. For spherical components, measurement is more complicated and labor intensive due to the constraint on axial location defined by the radius of curvature of the surface under test (SUT). Similarly to planar components, for spherical optics which are meniscus or near-meniscus shape factor, back reflections can cause measurement degradation and phase print through. Furthermore, the additional measurement of Radius of Curvature (RoC) often accompanies surface figure measurements which require two measurements: one at the cat's eye position (focus of transmission sphere (TS) located at the vertex of the SUT) and the confocal position (focus of the TS located at the RoC of the SUT). ${ }^{10}$ The distance between these two locations is recorded with some form of digital readout rail ${ }^{11}$ or laser tracker. ${ }^{12}$ Commercial instruments can regularly achieve $10 \mu \mathrm{m}$ level accuracies.

This conventional approach suffers from a number of drawbacks. It is encumbered by Abbe's error, i.e., error resulting from the distance between measurement loci being measured not along the optical axis, also it is labor intensive (in ideal case two measurements are required, in practice more) and requires an accurate distance measurement device such as encoder or DMI with an optical bench. All these reasons add up to measurement ambiguity and possibility of errors. SCI has an inherent ability to measure absolute values of OPD so the method presented here has a potential to provide accurate RoC readings from a single measurement performed at confocal position in addition to the surface figure.

\section{THEORY}

SCI exploits the relationship between spectral power density and the coherence function through the WienerKhinchin Theorem, ${ }^{13}$

$$
\Gamma(\tau)=\mathscr{F}\left[S\left(\nu-\nu_{0}\right)\right],
$$

where, $\Gamma$, is the coherence function, $S\left(\nu-n u_{0}\right)$, is the spectral power density, and the $\mathscr{F}$ operator is the Fourier Transform. Through controlled modulation of the source spectrum, additional coherence envelopes are produced at locations proportional to the modulation frequency, as a result of the Fourier Transform relationship,

$$
\mathscr{F}[f(\nu) \times \cos (f \nu)]=F(\tau)+F(\tau) \otimes \frac{1}{2}[\delta(\tau-f)+\delta(\tau+f)]=F(\tau)+\frac{1}{2} F(\tau-f)+\frac{1}{2} F(\tau+f) .
$$

The coherence function can be written in terms of wavelength and the measurement parameter, Optical Path Difference (OPD) assuming a Gaussian form of the nominal spectral distribution and cosine modulation implementation,

$$
\begin{aligned}
\gamma(O P D) & =\exp \left[-\left(\frac{\Lambda}{\lambda_{0}^{2}}\right)^{2} \times O P D^{2}\right] \\
& +\frac{1}{2} \exp \left[-\left(\frac{\Lambda}{\lambda_{0}^{2}}\right)^{2} \times\left(O P D-\frac{\lambda_{0}^{2}}{\Delta \lambda}\right)^{2}\right]+\frac{1}{2} \exp \left[-\left(\frac{\Lambda}{\lambda_{0}^{2}}\right)^{2} \times\left(O P D+\frac{\lambda_{0}^{2}}{\Delta \lambda}\right)^{2}\right],
\end{aligned}
$$

where, $\gamma$, is the normalized coherence function, $\Lambda$, is the source bandwidth, $\lambda_{0}$, is the central wavelength, and , $\Delta \lambda$, is the period of the modulation function. ${ }^{3}$

While the widths of the nominal coherence envelope and sideband envelopes are determined by the source bandwidth, the location of the sidebands is controlled explicitly by the control parameters of the modulation function at the fringe location, $l_{c}$,

$$
l_{c}=\frac{\lambda_{0}^{2}}{\Delta \lambda}
$$

These equations allow the formation and manipulation of localized, high contrast fringes in unequal path interferometers such as Fizeau configurations which benefit from common path implementations. If two surfaces in the measurement cavity are separated by a distance greater than the envelope width, their reflections can be adequately isolated for measurement. Surfaces as close as $50 \mu \mathrm{m}$ have been demonstrated with successful isolation. ${ }^{3}$ 


\section{SURFACE MEASUREMENTS}

\subsection{Flat Optics}

A $105 \mathrm{~mm}$ hypotenuse right angle prism was used to demonstrate achievable cavity lengths with current SCI methods. While extended cavity lengths are challenging from a design perspective, there is fundamentally no upper limit. The prism is measured in transmission against a transmission flat (TF) shown in Figure 1. In this

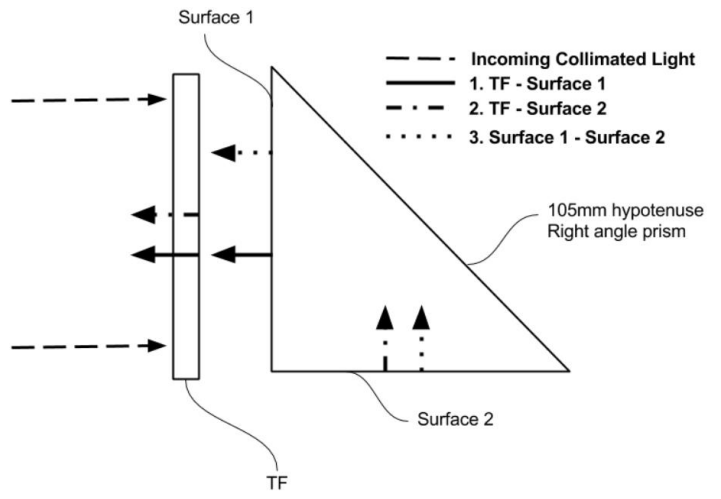

Figure 1. Right angle prism measurement setup. Each cavity is designated with a unique line type to demonstrate which cavities are present and between which surfaces. Each cavity can be isolated with SCI for measurement without the requirement of realignment or inducing intentional tilt to prevent spurious back reflections.

orientation, three interferograms can be generated and measured for all three cavities (TF and surface 1 , TF and surface 2, and surface 1 and surface 2) with no adjustment to the alignment of the SUT. Each cavity is independently isolated via proper tuning of the modulation frequency, such that, $l_{c}=d_{\text {cavity }}$. Because the cavity distance is an optical path length, refractive index must be accounted for. With this prism and measurement configuration we demonstrate measurements of cavities in excess of $150 \mathrm{~mm}$.

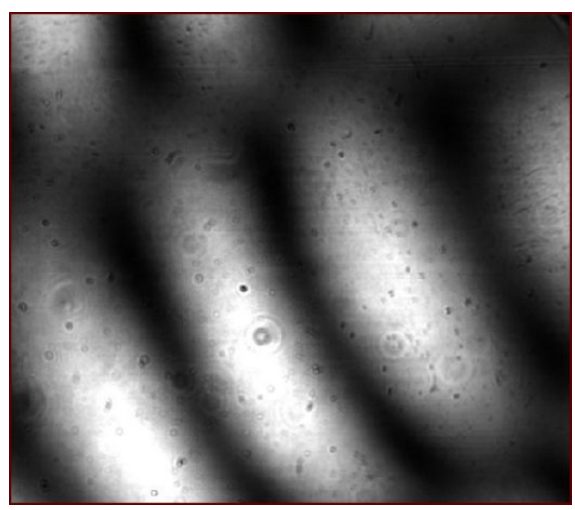

Figure 2. Interferogram of right angle prism when measured in coherent, 'alignment' mode of SCI Fizeau. While coherent mode is convenient for mechanical alignment of SUT, the long coherence length of the source precludes successful measurement of any one surface.

Such measurements for this optic are not possible with standard coherent interferometers due to back reflections shown in Figure 2 and a comparison between the SCI Fizeau and the Äpre Instruments S-100HR coherent Fizeau cannot be made. Figure 3 shows measured phase maps for each corresponding cavity with Tilt removed processed in Äpre Reveal ${ }^{\mathrm{TM}}$ metrology software.

In Figure 3, the left image is the phase map for the cavity between the TF and surface 1 of the right angle prism. The wavefront phase map has 0.413 waves peak to valley (PV) and 0.059 waves RMS. The center image 

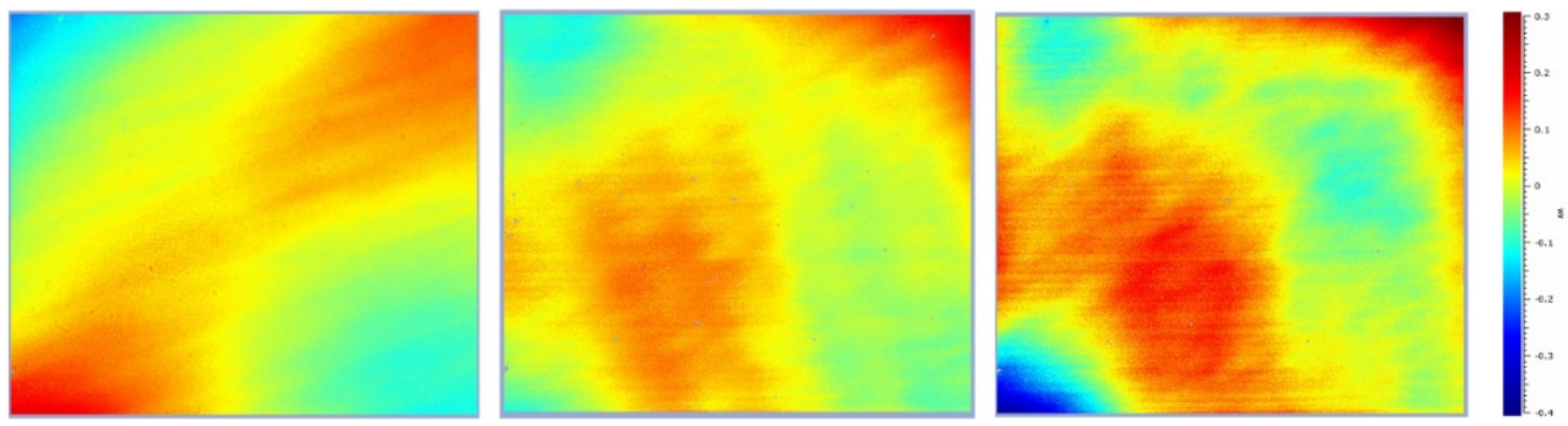

Figure 3. Right angle prism phase maps (image scale $\in[-0.4$ wvs, 0.3 wvs $]$ )Left: Phase map for cavity between TF and surface 1. Center: Phase map for cavity between TF and surface 2. Right: Phase map for cavity between surface 1 and surface 2 .

is the phase map for the cavity between the TF and surface 2 of the right angle prism. The wavefront phase map has 0.376 waves PV and 0.048 waves RMS. The right image is the phase map for the cavity between surface 1 and surface 2 of the right angle prism. The wavefront phase map has 0.794 waves PV and 0.074 waves RMS.

\subsection{Spherical Optics}

Spherical optical surfaces are both more labor intensive and difficult to measure with interferometers than flat optics. This is due to the increased constraint on axial location and centration of the SUT to the TS aperture. While commercial incoherent interferometers exist to meet the demands of localizing fringes in spherical surface cavities, these solutions increase the difficulty of alignment by a considerable amount by constraining the axial location of the lens to within tens of microns of the fringe location. SCI allows alignment of the components in the coherent mode operation and once the SUT is aligned, the SCI can be switched to incoherent mode on the time scale of the frame rate of the camera to remove contributions from any back reflections which may add coherently at the detector.
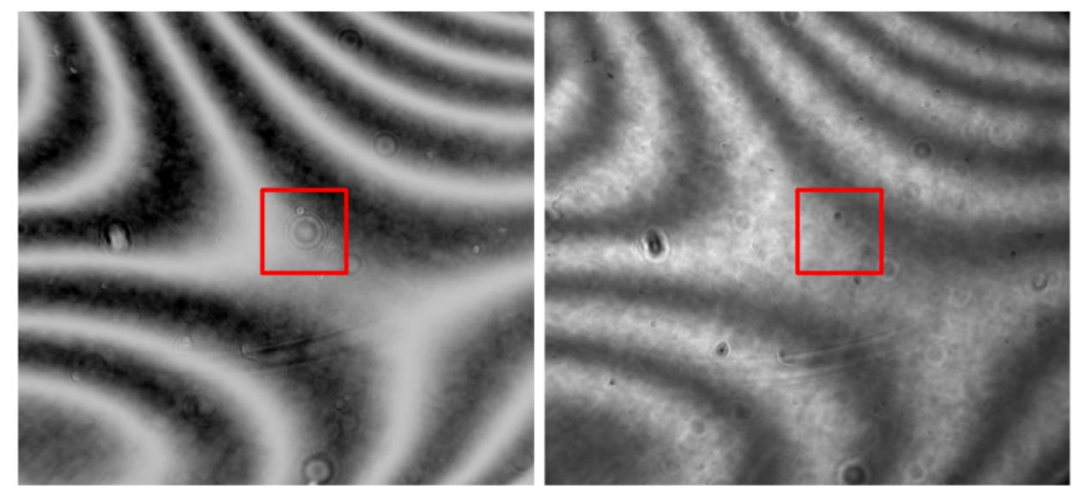

Figure 4. Left: Presence of coherent back reflection in coherent alignment mode of SCI Fizeau. Right: Incoherent measurement mode of SCI Fizeau localizes fringes at SUT and prevents temporally coherent contributions of secondary reflections at the detector plane.

Two different spherical lenses were measured on both the Äpre S-100HR and the SCI Fizeau interferometers. The first lens is a $60 \mathrm{~mm}$ diameter $444.4 \mathrm{~mm}$ focal length lens and the second lens is a $60 \mathrm{~mm}$ diameter $117.6 \mathrm{~mm}$ focal length lens, both of which are measured with a F/1.5 transmission sphere (TS). All phase maps were processed and analyzed in Äpre Reveal ${ }^{\mathrm{TM}}$ metrology software with Tilt and Power removed. While both lenses are near meniscus shape factor, the difference in the radii of curvatures of the front surfaces and the back surfaces does not cause significant back reflections or large scale measurement degradation. Figure 4 demonstrates the 
common problem of spurious back reflections in near-meniscus shape factor lenses with the presence of a small characteristic 'bullseye' pattern.

The left image of Figure 4 is an interferogram generated by the SCI Fizeau in coherent mode of the $444.4 \mathrm{~mm}$ focal length meniscus lens first surface. The return from the secondary surface is highlighted in the box. The right image of Figure 4 is the same interferogram but generated by the SCI Fizeau in incoherent mode for measurement. The display box is located in the same region of both images to demonstrate that the return from the secondary surface does not contribute to the incoherent interferogram.
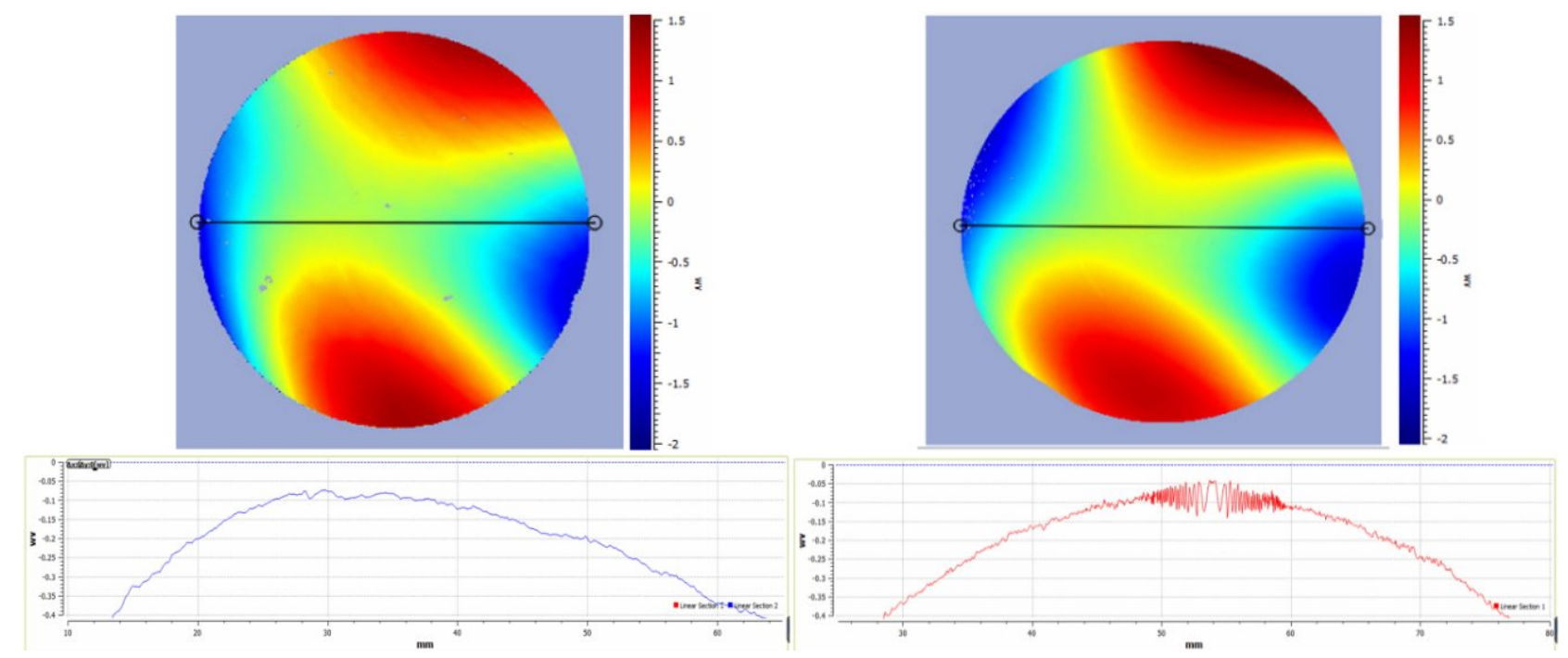

Figure 5. Left: SCI Fizeau phase map for the $444.4 \mathrm{~mm}$ focal length near-meniscus lens with x-axis section section to display absence of phase print through effects due to localized fringes in measurement space. Right: Äpre S-100HR phase map for the $444.4 \mathrm{~mm}$ focal length near-meniscus lens with y-slice section to display phase print through effects with 0.10 waves PV magnitude. Phase map image scale $\in$ [-2.0 wvs, 1.5 wvs]. X-axis section scale $\in[-0.4$ wvs, 0 wvs $]$

The resulting phase print through in the measurement of these surfaces is clearly visible in the $\mathrm{x}$-axis sections of the phase maps shown in Figure 5. For the phase map measured on the coherent interferometer, phase oscillations on the order of 0.1 waves PV are present in the center of the aperture, but are absent in SCI measurements. While the effects of this print through are not drastic due to the departure of the lens's shape factor from true meniscus, this effect can be strong enough in some cases to prevent measurement.

In Figure 5, the phase map on the left is measured on the SCI Fizeau. The phase map has 4.58 waves PV and 0.62 waves RMS. The phase map on the right is measured with Äpre S-100HR. The phase map has 3.50 waves $\mathrm{PV}$ and 0.69 waves RMS. The two measurements exhibit normal departure in the RMS metric due to potentially small variations in the measured apertures of the SUT.

In Figure 6, the phase map on the left is measured on the SCI Fizeau. The phase map has 1.12 waves PV and 0.09 waves RMS. The phase map on the right is measured with $\ddot{A}$ pre S-100HR. The phase map has 0.64 waves PV and 0.10 waves RMS. The two measurements show very good agreement in RMS Wavefront error establishing high quality measurement capability with the SCI method for measuring spherical optics.

\section{MEASUREMENT OF RADIUS OF CURVATURE WITH SCI}

SCI has an inherent ability to measure absolute optical path lengths and can be successfully employed to measure quantities such as ROC or thickness of the optical element solely by controlling the parameters of spectral modulation. Equation 4 outlines physical principle behind the distance measurement capability. Knowledge of both mean wavelength of the source and the modulation period determines in absolute terms the location of interference fringes. The distance measurement in some aspects resembles operation of white-light scanning 

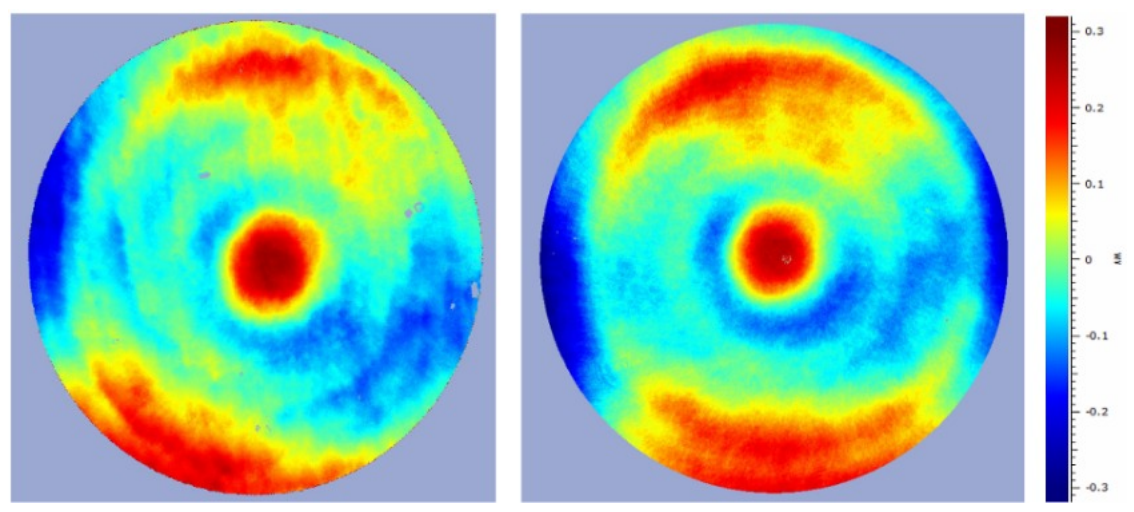

Figure 6. Phase maps for $117.6 \mathrm{~mm}$ focal length near-meniscus lens (image scale $\in$ [-0.3 wvs, 0.3 wvs]). Left: Phase map measured with SCI Fizeau. Right: Phase map measured with Äpre S-100HR.

interferometry (WLI) because the distance is established by determining the peak of coherence envelope formed by interference. To that effect, several frames are acquired in sequence for spectral modulations corresponding to different fringe distances and data is analyzed for maximum of fringe contrast. The resulting location of the best visibility fringes corresponds to the distance of the measured surface from the reference.

The reference surface can be a transmission flat, front surface of a prism or reference surface in a transmission sphere. In case of the latter, ROC of the measured surface can be calculated by subtracting this value from the nominal ROC of the reference surface. The outlined method is particularly attractive because the measurement of ROC and surface figure can be done in a single setup. Furthermore, no means of distance measurements are necessary such as encoders or DMI which can add cost to the setup. Also, the measurement by definition is performed on axis with no Abbe's error.

This is an active area of research and preliminary studies are showing promising results for simplifying radius of curvature measurements of spherical optics through the use of SCI. For accurate measurement and reliable phase reconstruction, stable control over both mean wavelength, $\lambda_{0}$, (as in other interferometry modalities) and the modulation period, $\Delta \lambda$, shown in Equation 4, is required to maintain both proper phase relationship and constant fringe contrast over the measurement cycle.

\section{CONCLUSION}

Spectrally controlled interferometry offers distinct advantages over traditional interferometry methods for both spherical and flat optical surfaces. In both cases, back reflections can decrease the accuracy of measurements and even prevent measurements entirely. SCI offers the advantage of alignment of the SUT in coherent mode which reduces the alignment time by orders of magnitude when compared to entirely incoherent interferometers. For measurement acquisition, SCI produces incoherent, localized fringes which provide complete surface isolation and fringe location is controlled without mechanical path length matching apparatus. Because the control parameters for SCI are implemented at the source level of the instrument, upgrading existing systems to utilize SCI methods can be simple. Measurement of flat optical cavities have been demonstrated with lengths in excess of $150 \mathrm{~mm}$. SCI methods have been demonstrated for measuring optical cavities with lengths over 3 orders of magnitude while still preserving measurement accuracy and surface isolation. Furthermore, measurement of spherical cavities have been demonstrated and compared with coherent interferometer results with good agreement. SCI has the potential to provide accurate $\mathrm{RoC}$ and surface figure measurements in a single measurement while also reducing measurement complexity, cost, and time when compared to existing methods.

\section{ACKNOWLEDGMENTS}

The authors would like to thank Dr. Jim Schwiegerling for useful technical discussion and the College of Optical Sciences at the University of Arizona for supplying optical lenses for testing. 


\section{REFERENCES}

[1] Malacara, D., [Optical Shop Testing], Wiley series in pure and applied optics, John Wiley \& Sons, Inc., Hoboken, NJ, USA (jun 2007).

[2] Wyant, J. C., "White light interferometry," 4737, 98-107, International Society for Optics and Photonics (jul 2002).

[3] Salsbury, C. and Olszak, A., "Thin Optical Window Measurement with a Spectrally Controlled Interferometer," in [Am. Soc. Precis. Eng. Top. Meet.], American Society of Precision Engineers, Tucson (2017).

[4] Kozhevatov, I. E. and Kulikova, E. K., "Interferometric Methods for Surface Testing. High-Order WhiteLight Interferometer," Instruments Exp. Tech. 44(1), 84-87 (2001).

[5] Kozhevatov, I. E., Rudenchik, E. A., Cheragin, N. P., and Kulikova, E. H., "A new in situ method for testing the optical thickness of removed transparent elements," in [Curr. Res. Hologr. Interferom. Methods Meas. Object Prop.], Zakharov, Y. N., ed., 50, International Society for Optics and Photonics (sep 2003).

[6] Kozhevatov, I. E. and Kulikova, E. K., "Higher-order Wideband Optical Interferometry," Radiophys. Quantum Electron. 46(1) (2003).

[7] Schwider, J., "White-light Fizeau interferometer," Appl. Opt. 36(7), 1433-1437 (1997).

[8] Schwider, J., "Coarse frequency comb interferometry," in [Interferom. XIV Tech. Anal.], Schmit, J., Creath, K., and Towers, C. E., eds., 706304, International Society for Optics and Photonics (aug 2008).

[9] Ai, C. and Wyant, J. C., "Effect of spurious reflection on phase shift interferometry," Appl. Opt. 27, 3039 (jul 1988).

[10] Selberg, L. A., "Radius measurement by interferometry," Opt. Eng. 31, 1961 (sep 1992).

[11] Schmitz, T. L., Gardner, N., Vaughn, M., Medicus, K., and Davies, A., "Improving optical bench radius measurements using stage error motion data," Appl. Opt. 47, 6692 (dec 2008).

[12] Zhao, C., Zehnder, R., and Burge, J. H., "Measuring the radius of curvature of a spheri- cal mirror with an interferometer and a laser tracker," Opt. Eng. 44(9) (2005).

[13] Born, M. and Wolf, E., [Principles of Optics: Electromagnetic Theory of Propagation, Interference and Diffraction of Light], Cambridge, 7 ed. (1980). 